\title{
DES SECRETS SOUS LES DÉCOMBRES : LA (DIS)SIMULATION DANS LE ROMAN POST-SISMIQUE HAÏTIEN
}

\author{
Alessia ViGNOLI \\ Université de Varsovie
}

\begin{abstract}
En): In January 2010 a major earthquake strikes Haiti, especially its capital Port-auPrince, and its consequences are still shaping Haitian life, politics and culture. The works of fiction published in the aftermath, from 2010 until today, are a vivid example of the intrusion of catastrophic events into literary production. The aim of this paper is to investigate the dynamics between simulation and dissimulation in two novels published after the earthquake: Aux frontières de la soif (2012) by Kettly Mars and L'escalier de mes désillusions (2014) by Gary Victor. In Mars's novel the main character keeps a horrible secret and makes many efforts in order not to reveal it to his friends and family, showing all the contradictions of everyday life in the post-catastrophic Port-au-Prince. In Victor's novel, the function of the apocalyptic event is to awaken some forgotten memories of the past which brutally reappear in the present. By analyzing these two novels, we will focus on the role of (dis)simulation as a survival strategy adopted by the characters to go on with their lives.
\end{abstract}

Keywords (En) : post-earthquake literature ; natural disaster ; Port-au-Prince ; Kettly Mars ; Gary Victor ; survival ; repression

Mots-clés (Fr) : littérature post-sismique ; catastrophe naturelle ; Port-au-Prince ; Kettly Mars ; Gary Victor ; survie ; refoulement

\section{Introduction}

Le 12 janvier 2010, la capitale haïtienne Port-au-Prince est ravagée par un tremblement de terre dévastateur, dont les conséquences socio-économiques, politiques et culturelles sont évidentes encore aujourd'hui. Cet événement catastrophique a aussi entraîné des bouleversements remarquables dans le champ littéraire haïtien. Force est de constater l'impact de la catastrophe naturelle sur la production littéraire: la littérature haïtienne contemporaine est en effet profondément influencée par la mise en fiction du tremblement de terre. Le corpus post-sismique, déjà plutôt ample, rassemble des ouvrages de tout genre qui ont paru à partir de 2010. Plusieurs questions concernant de façon plus ou moins directe l'écriture de la catastrophe sont traitées dans ce corpus hétérogène en évolution constante, qui pourrait être défini, selon l'expression utilisée par le psychiatre et intellectuel haïtien-québécois Joël DES RosIERS (2013 : 137), comme une littérature « rassemblée autour de la faille $»^{1}$.

1 Parmi les ouvrages appartenant à ce corpus figurent des poèmes, pièces de théâtre, essais, reportages, recueils de nouvelles et romans. Des auteurs de renommée internationale comme Dany Laferrière (Tout bouge autour de moi, Montréal, Mémoire d'encrier, 2010) et Yanick Lahens (Failles, Paris, Sabine Wespieser Éditeur, 2010) ont choisi une forme hybride entre chronique et essai alors que d'autres, comme Louis-Philippe Dalembert (Ballade d'un amour inachevé, Paris, Mercure de France, 2013), Kettly Mars et Gary Victor, se sont tournés vers la fiction. De plus, une nouvelle génération de romanciers est née après le tremblement de terre, à laquelle appartiennent 
Nous focaliserons notre attention sur deux romans qui appartiennent à la littérature haïtienne contemporaine, publiés après le séisme, pour illustrer la fonction des mécanismes de simulation et de dissimulation à l'intérieur de l'intrigue romanesque. Les ouvrages qui composent notre corpus d'étude sont Aux frontières de la soif de Kettly Mars, publié d'abord à Port-au-Prince en 2012 et ensuite à Paris, aux Éditions Mercure de France, en 2013, et L'escalier de mes désillusions de Gary Victor, publié en 2014 à Paris, aux Éditions Philippe Rey. L'analyse des deux romans montrera que la dissimulation ${ }^{2}$ et la simulation ${ }^{3}$ permettent aux personnages d'oublier certains épisodes du passé ou du présent, pour essayer de survivre dans des circonstances souvent insupportables.

\section{Secrets morbides et reconstruction d'une conscience dans Aux frontières de la soif de Kettly Mars}

Kettly Mars, née à Port-au-Prince en 1958, a publié plusieurs poèmes, nouvelles et romans, parmi lesquels L'heure hybride (2005), Fado (2008) et Saisons sauvages (2010). Particulièrement intéressée à la critique des mœurs, l'auteure présente dans ses ouvrages des « personnages composites, qui tentent de trouver leur place au sein d'une société qui bien souvent les ignore, ou bien les rejette » (CHEMLA, 2015 : 198). En 2010, au cours d'un entretien, Kettly Mars a souligné l'importance de l'écriture et du rôle de l'écrivain après la catastrophe : « Le 12 janvier m'a appris encore un peu plus le pouvoir de la parole. Les auteurs et les intellectuels haïtiens ont été les premiers, avant le gouvernement, à s'exprimer d'Haïti. » (ANDRIAMIRADO, 2010). Elle a pourtant attendu jusqu'en 2013 pour publier son premier ouvrage sur le tremblement de terre, Aux frontières de la soif, dédié « aux survivants du séisme du 12 janvier 2010 », auquel fait suite un autre roman situé à l'époque post-sismique, Je suis vivant, publié en 2015 à Paris, aux éditions Mercure de France. Dans Aux frontières de la soif, l'auteure confirme son intérêt profond pour la représentation de la réalité haïtienne et de ses contrastes, et elle proclame en même temps la force de ce «pouvoir de la parole », symbolisé par l'écriture, qui est le seul salut dans une société dégradée. Il est important de souligner à cet égard que l'un des thèmes fondamentaux du roman est l'éternel questionnement sur la "place de l'écrivain et de l'écriture dans cette désagrégation continue de la société » (CHEMLA, 2015 : 197).

\subsection{Une paralyse individuelle et sociale, une reconstruction impossible}

Aux frontières de la soif se déroule dans un univers post-catastrophique où la puissance créatrice de l'écriture et sa valeur rédemptrice sont associées à une vocation à la destruction et à l'anéantissement de soi, incarnée par la conduite

Marvin Victor (Corps mêlés, Paris, Gallimard, 2011), Makenzy Orcel (Les Immortelles, Paris, Zulma, 2010) et James Noël (Belle merveille, Paris, Zulma, 2017).

2 Selon la définition de Jean-Pierre CavaiLle (2009: 91), «[...] maintenir une chose secrète en faisant comme si elle n'existait pas ».

$3 \ll[\ldots]$ feindre que quelque chose existe qui n'est pas » (CAVAILLE, $2009: 91$ ). 
immorale du personnage principal du récit. Comme l'a constaté Martin Munro dans son étude consacrée à la littérature haïtienne post-sismique, le roman aborde le thème du lien étroit entre deux pulsions opposées, la création et la destruction, que le critique considère comme un effet paradoxal du tremblement de terre ${ }^{4}$. Dès les premières pages, la réalité post-sismique haïtienne est illustrée dans sa dimension la plus tragique à travers la description de Canaan, un immense camp de réfugiés devenu un véritable bidonville de plus de 80.000 personnes «qui était à lui seul un microcosme de la situation post-séisme du pays » (MARS, 2013 : 99). La corruption et la pauvreté règnent dans cet endroit infernal, dont l'appellation biblique renvoie paradoxalement à la terre promise par Dieu aux Hébreux. En explorant ce labyrinthe sans sortie où des mères et des pères sont contraints de vendre les corps de leurs enfants au plus offrant, Kettly Mars traite un sujet difficile et particulièrement délicat : la prostitution des mineurs dans les camps de réfugiés qui se sont constitués après le séisme. Dans l'enfer de Canaan tout est en vente, en particulier les drogues et le sexe.

Le roman commence un an après le tremblement de terre, un vendredi soir de janvier 2011, et se termine une dizaine de jours plus tard. Dans la narration linéaire s'insèrent des sauts en arrière, des anticipations dans lesquelles sont narrées les visites à Canaan d'un client habituel, Fito Belmar, personnage principal du roman. Fito, un quinquagénaire qui fréquente le camp une fois par semaine, le vendredi soir, est « un homme qui fuyait des spectres échappés de son impuissance et sa peur de vieillir, et cherchait refuge dans l'innocence, même s'il devait pour cela la profaner. » (MARS, 2013 : 83). L'impuissance de Fito se montre à plusieurs niveaux : d'abord, c'est une impuissance physique, car il réussit à soulager ses désirs seulement à travers une pratique sexuelle déviante, mais plus en général il est représenté comme un homme bloqué et paralysé, suite aux échecs qui ont caractérisé sa vie, et encore profondément choqué par le tremblement de terre. Du point de vue professionnel, Fito se partage entre son activité d'architecte urbaniste et celle d'écrivain. Un an après le séisme, il est au service d'une Organisation non gouvernementale et travaille pour la reconstruction du pays, une reconstruction presque impossible, car tout procède au ralenti et les actions douteuses des ONG révèlent toutes les contradictions de l'aide internationale. De plus, après un premier roman qui a remporté un grand succès, Fito est désormais un écrivain en panne d'inspiration qui craint de ne pas être à la hauteur des attentes de son éditeur et de son public. Du point de vue de sa vie privée, les échecs se succèdent : après deux divorces, Fito veut quitter sa compagne, Gaëlle, qui attend depuis six ans une demande en mariage. Cet homme ambigu et malheureux est donc coincé dans un état intermédiaire entre la vie et la mort, et il noie sa frustration dans l'alcool pour essayer d'oublier ses multiples déceptions, en compagnie de quelques morceaux de Miles Davis. La stagnation qui caractérise le personnage est le miroir d'une stagnation plus vaste, celle du pays; dans son roman, Kettly Mars insère des passages fortement critiques à l'égard de l'absence de l'État dans la gestion de

4 « [...] writing becomes for Mars's author-protagonist a fraught activity, related to his sexual exploitation of young girls, both of which activities are in turn related to the earthquake and its paradoxical effects : the ways in which it brings into sharp relief the close connection between the human impulses to create and destroy. » (MunRo, 2014 : 123). 
l'émergence après le séisme, des inégalités sociales et de l'ambiguïté de l'aide internationale, représentée par de nombreuses ONG et par l'ONU, accusée d'être le responsable de la diffusion du choléra dans le pays :

[...] l'épidémie de choléra qui tuait chaque jour des riverains du fleuve Artibonite et s'étendait rapidement aux autres départements du pays, la rumeur de plus en plus insistante affirmant que la maladie venait des excréments du contingent de casques bleus vivant au bord du fleuve et porteurs sains du bacille tueur. (MARS, $2013: 51$ ).

La situation post-catastrophique est certainement liée à la conduite de Fito, mais elle n'est pas à l'origine de ses déboires; en effet, les insuccès de cet individu frustré et impuissant ont commencé bien avant le séisme, l'on pourrait même affirmer qu'il profite de la situation de misère qui s'est créée dans les camps de réfugiés, vu que cette dégradation physique et morale lui permet de satisfaire ses instincts malsains. Fito, personnage autodestructeur tombé dans la déchéance ${ }^{5}$, entreprend un parcours de rédemption qui, à la fin du roman, l'amène à une délivrance inattendue à travers la pratique de l'écriture. Ce tournant dans l'existence de l'architecte-écrivain est marqué par l'arrivée de Tatsumi, une femme japonaise d'une quarantaine d'années, journaliste et professeure de littérature francophone, venue en Haïti pour écrire un article. Pendant les quelques jours passés en compagnie de Tatsumi aux Abricots, un village de pêcheurs dans le sud-ouest du pays, Fito entame un itinéraire de reconstruction de sa propre conscience, dans la tentative de se défaire des souillures du passé.

\subsection{De la (dis)simulation à une rédemption possible}

Dans Aux frontières de la soif, Kettly Mars confie la narration à des voix plurielles, ce qui permet au lecteur de saisir les multiples aspects de la situation post-séisme: au narrateur omniscient fait suite une focalisation interne à la troisième personne quand il s'agit des séquences qui concernent les visites de Fito à Canaan, ou bien à la première personne dans le cas des monologues intérieurs de quelques enfants et d'une femme du camp. Une analyse plus approfondie de la conduite de Fito permet d'observer l'ambivalence de ce personnage, qui vit une existence double et ne parle à personne de ses troubles psychiques, symptômes d'un malaise plus vaste, très probablement un syndrome de stress posttraumatique. Fito est décrit dès le début du roman comme un homme qui dissimule et masque la vérité : «Dans ses courriels à Tatsumi, Fito avait oublié de lui parler de Gaëlle, comme il avait oublié de mentionner d'autres choses de sa vie. Comme Canaan. » (MARS, 2013 : 25) ; «Il ne s'était pas encore confié à Franck ni aux autres à propos de ses virées à Canaan. » (MARS, 2013 : 47). Fito garde son secret inavouable, conscient de la gravité de ses actions ; pris de remords après une de ses visites à Canaan, il prend rendez-vous avec une psychologue clinicienne dans l'espoir de «sauver son âme» (MARS, 2013: 134) en s'ouvrant à une professionnelle. Bloqué encore une fois face à la possibilité de sortir de sa

5 Fito Belmar est décrit comme suit par MUNRO (2014 : 133) : « [...] a flawed, self-destructive writer figure who appears powerless to stop his descent into complete dissolution. ». 
situation sans issue, il renonce à cette rencontre, convaincu qu'une femme ne pourrait pas comprendre ses troubles sans le considérer comme un monstre: «À mesure que les heures passaient, l'idée d'une session avec une psychologue lui déplaisait de plus en plus. [...] Il lui fallait un homme, un mâle comme lui, ressentant dans sa chair les pulsions et les exigences de leur sexe » (MARS, 2013: 135). Dans son entourage, Franck est le seul à exprimer ouvertement ses doutes. Quand il demande à son ami la raison de ses visites à Canaan, Fito est confronté à la réalité :

Il ressentit pour la première fois depuis qu'il allait à Canaan un sentiment qu'il identifia clairement, qu'il fuyait mais qui le cerna et le brûla, la honte. Tout ce temps qu'ils se parlaient, Franck était au courant. Comment savait-il ? Depuis combien de temps savait-il ? (MARS, 2013 : 78).

Malgré cette révélation, le secret de Fito demeure caché et ronge sa conscience, ou ce qu'il en reste : "Quels démons habitaient ce corps qui gardait encore un air d'adolescence malgré les fêlures de l'âge ? Quel pouvait être la cause secrète et sombre du tourment qui le rongeait ?» (MARS, 2013: 125); "Quel était ce poids qu'il portait et le rendait haïssable à ses propres yeux ?» (MARS, $2013: 130$ ).

Si Fito Belmar dissimule, plusieurs aspects de sa conduite relèvent aussi de la simulation. D'abord, il vit dans le mensonge, car il ment à sa compagne Gaëlle, à ses amis, et même à Tatsumi : "Il lui avait menti sans vergogne, se prétendait heureux, la reconstruction du pays allait bon train, tout baignait dans l'huile. » (MARS, 2013 : 30). En outre, son existence tourne autour d'un contraste évident entre son profil public, celui d'un architecte engagé dans la reconstruction de son pays après la catastrophe, et son profil privé, celui d'un homme méprisable en lutte contre ses démons. Fito porte un masque social en faisant semblant d'être une personne respectable et seulement une fois par semaine, quand il se rend à Canaan, il dévoile toutes les facettes de sa personnalité.

Même si Fito ne raconte pas son combat intérieur à Tatsumi, la femme japonaise incarne un rôle central dans son parcours de rédemption : «Il aurait juré que Tatsumi savait quelque chose, qu'elle avait attendu ce moment pour entrer dans le gouffre de sa vie, pour le mettre à nu. » (MARS, 2013: 159). Non seulement elle réveille chez l'homme un désir sexuel normal, orienté vers une personne adulte mais, de plus, elle l'encourage à écrire pour guérir de ses blessures. Le week-end passé avec Tatsumi à la mer marque un nouveau commencement pour Fito, qui renoue avec le paysage naturel loin de l'enfer de Port-au-Prince et de Canaan. Une fois rentré dans le chaos de la capitale, Fito s'assoit devant son ordinateur et tape le titre de son prochain roman, Aux frontières de la soif, conscient que ses descentes dans Canaan appartiennent désormais au passé. 


\section{Le retour du passé refoulé dans L'escalier de mes désillusions de Gary Victor}

Le romancier et dramaturge Gary Victor, né à Port-au-Prince en 1958, est l'auteur d'une œuvre importante qui rassemble jusqu'à maintenant plus d'une quinzaine de romans, parmi lesquels À l'angle des rues parallèles (2000) et Le Sang et la Mer (2010). Comme l'a souligné le critique Yves ChemLa (2015: 161), Gary Victor dans son œuvre

[...] interroge dans un même mouvement les conditions d'exercice du pouvoir en Haïti et leurs soubassements mythologiques, les marquages sociaux imprimés depuis le temps de la plantation, la place de l'écriture et de l'intellectuel dans les dispositifs de représentation de cette histoire complexe.

Considéré comme l'un des auteurs les plus appréciés par le lectorat haïtien ${ }^{6}$, il a élaboré dans ses ouvrages de fiction une réflexion composite sur le pouvoir et ses perversions, en intégrant l'histoire et les mythes d'Haiti.

Dans L'escalier de mes désillusions, à ces thématiques toujours présentes chez Gary Victor s'ajoute la problématique toute contemporaine du tremblement de terre et des effets de son irruption dans l'intrigue romanesque. L'auteur reprend des sujets déjà abordés dans Maudite éducation, roman en partie autobiographique paru en 2012 où est racontée l'adolescence d'un jeune haïtien, Carl Vausier, dans les années soixante-dix, sous la dictature de François Duvalier. Carl, devenu adulte et écrivain de profession, est aussi le personnage principal de L'escalier de mes désillusions, qui s'ouvre sur les instants après le séisme, «deux heures après les ruades assassines de la terre »(VICTOR, 2014 : 14). Profondément choqué mais encore en vie, Carl se précipite vers la maison où habitent sa belle-mère Man Hernande, son ex-femme Jézabel et sa fille Hanna. Il y trouve seulement sa bellemère et ne parvient pas à avoir des nouvelles de Jézabel et Hanna; lorsqu'il comprend qu'il les a peut-être perdues, Carl, complètement pétrifié, s'assoit à côté de sa belle-mère et ils attendent ensemble des nouvelles probablement tragiques.

\subsection{Des secousses révélatrices}

Le tremblement de terre est un événement qui rythme le récit avec ses nombreuses répliques et qui déclenche chez Carl, alter ego fictionnel de Gary Victor, un mécanisme de récupération de certains souvenirs refoulés au tréfonds de sa mémoire. L'agitation du sol et ses soubresauts incessants ouvrent une fracture qui était fermée depuis longtemps et permettent à Carl de revenir sur des épisodes de son passé. Le narrateur et personnage principal du roman, emporté par la colère de la terre, s'égare alors dans le tourbillon de ses pensées et dans l'évocation de quelques événements saillants de son existence, comme ses histoires d'amour, son mariage avec Jézabel, l'apprentissage de l'écriture et les funérailles de son père. Au fur et à mesure que les réflexions de Carl sur son passé

6 « Gary Victor est sans doute l'un des écrivains les plus lus en Haïti. »(CHEMLA, 2015 : 161). 
se succèdent, le lecteur découvre un univers de secrets et de mensonges qui étaient enfouis dans les recoins de la mémoire et refont surface à chaque nouvelle réplique. Dans son «délire post-apocalyptique » (FERMI, 2015), la conscience traumatisée de Carl dévoile une réalité complexe, souvent insaisissable, qui reflète le caractère ambigu de la vie pendant la dictature. La période de la présidence de François Duvalier (1957-1971), appelé dans le roman «Son Excellence le Président éternel », est évoquée par Gary Victor à travers une atmosphère de soupçon et de mystère. Par l'entremise de son alter ego fictif, l'écrivain n'hésite pas à critiquer ouvertement la société haïtienne, « engluée dans ses mythes, dans son mépris de l'humain, dans son imaginaire si corrompu qu'un croque-mort nasillard, puis son fils avaient pu mettre sous coupe réglée plusieurs millions d'âmes. » (VICTOR, 2014 : 79).

La dissimulation est très présente dans le texte et même mentionnée explicitement par le narrateur à plusieurs reprises : «Les funérailles sont un des rares moments où on ne peut pas se cacher, se dissimuler, fuir. » (VICTOR, 2014 : $19) ;$ « [...] cet univers ténébreux que nous tentions tous d'ignorer, de dissimuler, d'enfouir sous des strates de masques [...]»(VICTOR, 2014: 49); «[...] je garderai mes peines comme un avare dissimule ses sous. » (VICTOR, 2014 : 111). De plus, presque tous les personnages du roman sont des dissimulateurs et toutes les vérités cachées qui sortent de l'obscurité au fil de la lecture s'imbriquent les unes dans les autres, dans un dédale d'incertitudes où le lecteur peine à s'orienter.

\subsection{Un labyrinthe de mensonges et de non-dits}

Nous conduirons une analyse des différents aspects de la dissimulation dans L'escalier de mes désillusions, en prenant en considération les personnages qui dissimulent et les non-dits qui marquent leurs existences. L'un des secrets de Carl Vausier est strictement lié à un autre personnage, André, que Carl avait rencontré aux funérailles de son père ; à cette occasion, André s'était adressé à Carl pour prononcer des mots quelque peu énigmatiques : " "Gaston Paisible est mort dans mes bras. Il m'a fait jurer de garder le silence. Nous avons tous nos secrets, Carl." »(VICTOR, 2014 : 20). En suivant les pensées de Carl, qui évoquent un passé lointain, le lecteur découvre que Gaston Paisible était un poète, homosexuel comme André, chez lequel Carl se rendait pour apprendre à écrire et pour recevoir des conseils sur ses premiers écrits.

Gaston aussi avait un secret, car il était atteint d'un cancer incurable, et il en avait parlé à Carl : «Il m'apprit - j'étais la seule personne à qui il le disait souffrir d'un cancer. » (VICTOR, 2014 : 50) ; «Gaston m'avait supplié de ne révéler à personne le secret de son cancer. »(VICTOR, 2014 : 51). Gaston était prêt à dévoiler à Carl une autre vérité dissimulée : "Il voulait aussi me confier un secret qui changerait, certifiait-il, mon regard sur lui. » (VICTOR, 2014 : 50). Lors d'une de ses rencontres avec Gaston, Carl était tombé sous le charme du poète et, sans s'en rendre compte, il fut profondément séduit par cet homme charmant; dégoûté par le désir que Gaston avait été capable de susciter en lui, Carl avait enfoncé un coupe-papier dans les omoplates du poète. Pourtant, l'affaire mystérieuse que Gaston voulait révéler à Carl n'était pas son homosexualité, 
comme Carl le croyait, car le poète ne cachait pas son orientation sexuelle, malgré les préjugés dont il était victime. Son véritable secret était sa participation à la lutte menée clandestinement contre la dictature et il aurait voulu proposer à Carl de le joindre dans le combat. Gaston Paisible, grièvement blessé, mourut peu après l'agression de Carl, dans les bras d'André, à qui il demanda de ne pas raconter ce qui s'était passé, donc de ne pas dénoncer Carl.

Si André connaissait ce lourd secret de Carl, Carl connaissait un secret de l'autre, car André aussi avait tué un homme, son père. Lors de leur première rencontre, qui se situe chronologiquement avant les funérailles du père de Carl, André avait confessé son crime, en se libérant ainsi d'un poids énorme : " "Son secret était trop lourd pour ses épaules, impossible pourtant à partager, sauf pendant le temps d'une confession.” »(VICTOR, 2014: 47). Entre les deux assassins s'était donc instauré un pacte silencieux de respect réciproque, puisque « chacun était en possession du secret de l'autre. »(VICTOR, 2014 : 76).

La dissimulation caractérise également la relation entre Carl et son ex-femme Jézabel, un mariage échoué, profondément influencé par les ombres et les fantasmes du passé. Dans le présent de la narration, au cours de sa longue attente assis à côté de sa belle-mère, Carl réfléchit sur sa vie avec Jézabel, une femme froide et insaisissable, hantée par des souvenirs obscurs : «Elle a cette grande capacité de dissimuler ses sentiments, si bien que je me questionne encore sur ce qu'elle a vraiment éprouvé envers moi tout au cours de notre relation. » (VICTOR, 2014 : 15-16). Jézabel avait raconté à son mari un épisode étrange : la nuit de ses dix-sept ans, pendant qu'elle dormait, elle avait été victime d'un viol. Cet événement absurde et inexplicable, où l'imaginaire vaudou l'emporte sur la rationalité, n'est pas le seul non-dit concernant le passé de Jézabel, comme le constate Carl : «J'avais l'intuition que la vie de Jézabel cachait, peut-être même à son insu, un secret encore plus opaque que ce viol en songe qu'elle prétendait réel. » (VICTOR, 2014 : 109). Seulement à la fin du roman, des révélations faites au cours d'un dialogue entre Carl et sa belle-mère, Man Hernande, viennent éclairer certains aspects de la personnalité troublée de Jézabel. Carl apprend ainsi des secrets que Man Hernande s'était gardée de partager, le «poids d'un passé dont même sa fille n'a pas connaissance » (VICTOR , 2014 : 171) : avant de déménager à Port-au-Prince, Man Hernande s'appelait Célia et travaillait comme domestique. Une nuit, elle avait été violée par un des ennemis de la dictature, Pierre Carnoux, qu'elle avait ensuite dénoncé aux miliciens au service du Président, au lieu de l'abriter. Ce héros de l'opposition au pouvoir dictatorial, emprisonné, torturé et tué à cause de Man Hernande, était donc le père que Jézabel n'a jamais connu : «J'ai juré de ne vivre que pour elle et de ne jamais lui révéler la vérité sur sa naissance. » (VICTOR, $2014: 172$ ) ; «Elle ne doit jamais savoir. Elle a tout fait pour découvrir la vérité. » (VICTOR, 2014 : 173). Man Hernande revient aussi sur l'épisode mystérieux du viol subi par Jézabel et, sollicitée par la curiosité de Carl, elle révèle l'identité du responsable, qui n'était rien d'autre que le frère de Pierre Carnoux, Joseph, soupçonné de sorcellerie. Dans cette fin d'après-midi de janvier, Carl découvre peu à peu des détails du passé de Jézabel qui l'aident à déchiffrer et en partie justifier la conduite inexplicable de sa femme : 
Je comprends pourquoi Man Hernande tient tant à maintenir sa fille dans l'ignorance de ces faits. Il faut qu'elle parte avec son secret. Ce père que Jézabel a tant voulu auprès d'elle [...] resterait à jamais pour elle un insondable mystère. (VICTOR, 2014 : 179)

Carl, André, Gaston, Jézabel et Man Hernande ont altéré la vérité pour des raisons différentes mais poussés par le même instinct de survie. Les récits oubliés qui resurgissent et reviennent troubler la conscience bouleversée de Carl dans le décor post-apocalyptique du 12 janvier 2010 montrent que la pratique de la dissimulation a permis aux personnages du roman de survivre dans une société paralysée par la superstition, où règnent l'hypocrisie et la simulation.

\section{Conclusion}

À l'intérieur du corpus qui a fait l'objet de notre étude, la (dis)simulation symbolise un mécanisme de survie adopté par les personnages de manière consciente ou pas, mais les enjeux sont différents dans les deux romans analysés. Kettly Mars dans Aux frontières de la soif décrit le parcours de rédemption d'un homme qui ne peut pas dévoiler un secret obscur et moralement inacceptable : ses pulsions pédophiles. Le passé et le présent de Fito Belmar sont régis par des mensonges qui lui permettent de survivre dans un pays détruit, dépourvu des moyens nécessaires pour renaître après la catastrophe. Les efforts de Fito pour cacher la vérité et les nombreux non-dits qui caractérisent ses actions montrent les contradictions qui règnent à Port-au-Prince un an après le séisme. Dans L'escalier de mes désillusions, Gary Victor s'adonne à la critique des mœurs en faisant le portrait d'une société désabusée où la (dis)simulation est une règle de survie ; pour Carl Vausier comme pour les autres personnages, dissimuler signifie garder des secrets dangereux ou pénibles qui remontent à la surface dès les premières secousses, réveillés par les convulsions meurtrières de la terre.

\section{BIBLIOGRAPHIE}

ANDRIAMIRADO Virginie (2010), La voix des écrivains haïtiens, Africultures, http://africultures.com/la-voix-des-ecrivains-haitiens-9426/

CAVAILlE Jean-Pierre (2009), Taire, mentir, simuler, dissimuler... un long héritage, La lettre de l'enfance et de l'adolescence 75, p. 87-94.

ChEMLA Yves (2015), Littérature haïtienne 1980-2015, Delmas, C3 Éditions.

DES ROSIERs Joël (2013), Métaspora : essai sur les patries intimes, Montréal, Triptyque.

FERMI Elena (2015), Gary Victor, L'escalier de mes désillusions, Studi Francesi 175 (LIX/I), http://studifrancesi.revues.org/663

MARS Kettly (2013), Aux frontières de la soif, Paris, Mercure de France.

MUNRO Martin (2014), Writing on the fault line. Haitian literature and the earthquake of 2010, Liverpool, Liverpool University Press.

Pessini Alba (2015), Kettly Mars, Aux frontières de la soif, Studi Francesi 171 (LVII/III), http://studifrancesi.revues.org/2919

VICTOR Gary (2014), L'escalier de mes désillusions, Paris, Philippe Rey. 